\title{
Consistent Quantization of Noncommutative D-brane in Non-constant B Field Background
}

\author{
Jen-Chi Lee* \\ Department of Electrophysics, National Chiao-Tung University, Hsinchu, \\ 30050, Taiwan
}

\begin{abstract}
We perform the consistent quantization of open string D-brane in nonconstant NS-NS closed string B field background by directly imposing the worldsheet conformal symmetry. In addition to the previous noncommutative D-brane coordinates quantization with constant noncommutative parameter $\theta$, we obtain a set of constraints on the B field which can be interpreted as a quantum consistent deformation of a curved noncommutative D-brane.

Noncommutative, D-brane.
\end{abstract}

Typeset using REVTEX

*e-mail: jcclee@cc.nctu.edu.tw 


\section{Introduction}

Historically, consistent quantization of string theory gave a remarkable result that the embedding spacetime dimension is restricted to $\mathrm{D}=26$. It was later realized in 80 s that this is equivalent to requiring the conformal symmetry on the worldsheet in the presence of target space flat metric $\eta$ background. For the last few years, it has been pointed out that when a constant NS-NS B field background is turned on on the D-brane, consistent quantization requires, in addition to $\mathrm{D}=26$, the noncommutativity of spacetime coordinates on the D-brane [1]. From the worldsheet conformal symmetry point of view, this is a new exact ( all orders in $\alpha^{\prime}$ ) fixed point of the theory corresponding to flat metric $\eta$ and constant B background. In the so-called Seiberg-Witten limit [2], Seiberg and Witten showed that all small perturbation calculation around this new fixed point, including the effective field theory and correlation functions etc. are dictated by replacing the ordinary operator product expansion, which has been used in the "old fixed point", by the Moyal star-product. For example, noncommutative Yang-Mills theory naturally appears in the low energy description of D-brane with constant B background in the Seiberg-Witten limit.

In this brief report, we consider the quantization of D-brane in the non-constant but slowly varying B background. In this case, it is obvious that the conformal symmetry or quantum consistency will be spoiled and one needs to modify the quantization. There are two possible strategies to restore the quantum consistency. One is to generalize the Moyal bracket to more complicated types. The other one is to put constraint on the B background but preserve the Moyal bracket quantization. Previous approach on this subject has been based on the first method [3]. The authors of [3] found that, in the case of vanishing field strength $\mathrm{H}=\mathrm{dB}=0$, one get the same noncommutative Moyal structure but with nonconstant $\theta$. With the generic nonvanishing $\mathrm{H} \neq 0$ case, one gets a more general noncommutative space with $\theta$ depending on $\mathrm{X}$ and $\mathrm{P}$. However, their treatment is valid only at the Poisson level, thus the quantum consistency is still not guaranteed and it is not clear whether the resulting 
theory is a consistent string theory. We will adopt the second approach in this paper and impose the conformal symmetry on the worldsheet by directly calculating the $\mathrm{T} \cdot \mathrm{T}$ algebra and obtain a set of constraints on B field. In contrast to the previous calculation which is valid only at Poisson level, our result here is an exact consistent quantization and thus resulting a consistent string theory.

The worldsheet action of an open string ending on a Dp-brane is

$$
S=\frac{1}{4 \pi \alpha^{\prime}} \int_{\Sigma} d^{2} \sigma\left[g^{\alpha \beta} \eta_{\mu \nu} \partial_{\alpha} X^{\mu} \partial_{\beta} X^{\nu}+\epsilon^{\alpha \beta} B_{\mu \nu} \partial_{\alpha} X^{\mu} \partial_{\beta} X^{\nu}\right]+\frac{1}{2 \pi \alpha^{\prime}} \oint_{\partial \Sigma} d \tau A_{i} \partial_{\tau} X^{i}
$$

where $A_{i}, i=0,1, \cdots, p$, is the $\mathrm{U}(1)$ gauge field on the Dp-brane. We will consider the case that both ends of the string are attaching to the same Dp-brane, and B is turned on only on the Dp-brane. In this case, the action in eq(1) can be rewritten as

$$
S=\frac{1}{4 \pi \alpha^{\prime}} \int d^{2} \sigma\left[g^{\alpha \beta} \eta_{\mu \nu} \partial_{\alpha} X^{\mu} \partial_{\beta} X^{\nu}+\epsilon^{\alpha \beta} F_{i j} \partial_{\alpha} X^{i} \partial_{\beta} X^{j}\right]
$$

where $\mathrm{F}=\mathrm{B}-\mathrm{dA}$. Note that $\mathrm{F}$ is invariant under the usual $\mathrm{U}(1)$ gauge transformation of $\mathrm{A}$ and the following transformation

$$
B \rightarrow B+d \Lambda, A \rightarrow A+\Lambda
$$

\section{Constant B Background}

We first consider the case $\mathrm{B}=\mathrm{B}^{(0)}=$ constant and $\mathrm{A}=0$. The worldsheet equation of motion of eq(2) with prescribed boundary conditions can still be solved exactly as in the B $=0$ case, and one can calculate the energy momentum tensor to be

$$
T_{++}=\frac{1}{2} \eta_{\mu \nu} \partial_{+} X^{\mu} \partial_{+} X^{\nu}=\frac{1}{2} M_{i j} \partial_{+} \widetilde{X^{i}} \partial_{+} \widetilde{X^{j}}+\frac{1}{2} \eta_{a b} \partial_{+} X^{a} \partial_{+} X^{b}
$$

where $a=p+1, \cdots, 9$, and a similar formula for $T_{--}$. Note that the region of worldsheet

space coordinate of both $T$ is $0 \leq \sigma \leq \pi$.In equation (4), $\left.\widetilde{X^{i}} \equiv X^{i}\right|_{F=0}$ and the effective open string metric [2] is

$$
M_{i j}=\eta_{i j}-B_{i k}^{(0)} \eta^{k l} B_{l j}^{(0)} .
$$


It is important to note that even in the presence of constant $\mathrm{B}^{(0)}$ background, one still has the following open string continuing relation

$$
X^{\prime}(\sigma)=-X^{\prime}(-\sigma)
$$

as in the case of $\mathrm{B}=0$ background $\mathbb{4}$. This enables one to continue the definition of $T_{++}$ to the region $-\pi \leq \sigma \leq \pi$ and to eliminate $T_{--}$. The conformal algebra of the Virasoro operator $L_{n}$ can thus be, as in the case of $\mathrm{B}=0$, translated to a single $T \cdot T$ algebra when one conformally maps the worldsheet from the cylinder to the complex plane. Hereafter we will neglect the irrelevant second term in equation (4) and refer $X^{i}$ to $\widetilde{X^{i}}, T$ to the continuing $T_{++} \cdot$

The quantization of the system was done by several groups [1]. One way to do it is to use an old formula of propagator of the action in eq (1) [5], and evaluate it at worldsheet boundary [2], one gets

$$
\left\langle X_{i}(\tau) X_{j}\left(\tau^{\top}\right)\right\rangle=-M_{i j} \log \left(\tau-\tau^{2}\right)^{2}+\frac{i}{2} \theta_{i j} \epsilon\left(\tau-\tau^{\top}\right)
$$

where $\epsilon(\tau)$ is the function that is 1 or -1 for positive or negative $\tau$, and

$$
\theta_{i j}=-2 \pi\left(M^{-1} F\right)_{i j}
$$

The original calculation [5] of the full propagator was done by the standard background field method with Riemann normal coordinate expansion, and was considered to be a stringloop effect since there is no closed string B field at open string-tree diagram without Dbrane.With the introduction of D-brane [6] into the theory, the propagator becomes an important string-tree effect.One remarkable observation of eq (7) is that the D-brane coordinates become noncommutative

$$
\left[X^{i}(\tau), X^{i}(\tau)\right]=i \theta^{i j}
$$

This result is not surprising from quantum Hall theory point of view, where the projective representation of magnetic translation group and the Moyal realization of $W_{1+\infty}$ symmetry 
[7] were discussed some time ago. We are now in a position to check the worldsheet conformal symmetry by calculating the $T \cdot T$ algebra. The calculation in the bulk is the same as in the free case. A direct calculation on the boundary gives the right conformal algebra

$$
T(\tau) \cdot T(\tau) \sim \frac{1}{2} \frac{M_{i j} M^{i j}}{(\tau-\tau)^{4}}+\frac{2}{(\tau-\tau)^{2}} \frac{1}{2} M_{i j} \partial_{\tau^{\prime}} X^{i} \partial_{\tau^{\prime}} X^{j}+\frac{1}{(\tau-\tau)} \partial_{\tau}\left(\frac{1}{2} M_{i j} \partial_{\tau^{\prime}} X^{i} \partial_{\tau^{\prime}} X^{j}\right)+\cdots
$$

Note that the first term on the r.h.s. is the same as in the $\mathrm{B}=0$ case, $M_{i j} M^{i j}=$ $d$ =spacetime dimension, and will be cancelled by the conformal ghost. It is now clear that the propagator in eq (7) and eq (4) is a consistent quantization. One notes that

turning on the constant $\mathrm{B}^{(0)}$ field means $\eta_{i j} \rightarrow M_{i j}$ and thus simultaneously turning on the noncommutative parameter $\theta$.

III. Non-constant B Background

We now turn to the non-constant B background.We will assume the weak or slowly varying B field

$$
B=B^{(0)}+b(X),
$$

where $B^{(0)}$ is a constant and $b(X)$ is a slowly varying field. In this case, the worldsheet equation of motion of $X^{i}$ can not be exactly solved and eq (6) no longer holds. It turns out not possible for generic $b$ to write down $T(\tau)$ with finite number of terms to close the $T \cdot T$ algebra. The treatment of [3] was to modify eq (9) at Poisson level, but they did not check the most important worldsheet conformal symmetry. To avoid this difficulty, we will further assume $H \equiv d b=0$ and trade $b$ to $d A$ by using eq (3). Since B is not turned on in the bulk, the $T \cdot T$ algebra is still valid there. We then propose the following boundary $T(\tau)$

$$
T(\tau)=\frac{1}{2} M_{i j} \partial_{\tau} X^{i} \partial_{\tau} X^{j}+M_{i j} A^{i} \partial_{\tau} X^{j}
$$

where the open string metric $M_{i j}$ is due to $B^{(0)}$ only.This form of $A^{i}$ coupling is consistent with worldsheet vertex operator consideration. We then check the worldsheet conformal symmetry by calculating the $T \cdot T$ algebra and neglect the second order $A^{i}$ term 


$$
\begin{aligned}
T(\tau) \cdot T(\tau) \sim & \frac{1}{2} \frac{d}{(\tau-\tau)^{4}}+\frac{2}{\left(\tau-\tau^{2}\right)^{2}}\left(\frac{1}{2} M_{i j} \partial_{\tau^{\prime}} X^{i} \partial_{\tau^{\prime}} X^{j}+M_{i j} A^{i} \partial_{\tau^{\prime}} X^{j}\right)+ \\
& \frac{1}{(\tau-\tau)} \partial_{\tau^{\prime}}\left(\frac{1}{2} M_{i j} \partial_{\tau^{\prime}} X^{i} \partial_{\tau^{\prime}} X^{j}+M_{i j} A^{i} \partial_{\tau^{\prime}} X^{j}\right)+ \\
& \frac{2}{(\tau-\tau)^{3}} M_{i j} \partial^{(j} A^{i)}+\frac{1}{(\tau-\tau)^{2}} M_{i j} \partial^{i} \partial^{j} A_{k} \partial_{\tau^{\prime}} X^{k} .
\end{aligned}
$$

The worldsheet conformal symmetry requires the following constraints on the background field A

$$
M_{i j} \partial^{(j} A^{i)}=0, M_{i j} \partial^{i} \partial^{j} A_{k}=0 .
$$

The constraints on the background A can then be traded back to the background $b$ to be

$$
M_{i j} \partial^{j} b^{i k}=0, M_{i j} \partial^{i} \partial^{j} b_{k l}=0 .
$$

In conclusion, the consistent quantization of D-brane in the presence of non-constant, slowly-varying background $\mathrm{B}$ in eq (11) with vanishing field strength $H \equiv d B=0$ requires constraints in eq (15) on the B field. Equation (14) is consistent with the equation of motion for noncommutative Yang-Mills in the weak field approximation. Equation (15) can be interpreted as a quantum consistent deformation of noncommutative D-brane by background B field, and is presumably related to a curved noncommutative D-brane characteristized by a $\mathrm{X}$ dependent $\theta(X)$. This is consistent with ref[3] although our treatment here is beyond the Poisson level. One expects that a modified associated star-algebra can be constructed on such a new curved noncommutative $\mathrm{D}$-brane. It seems that for the more generic $\mathrm{H} \neq 0$ case, one needs to introduce a nonassocitaed star-algebra to incorporate the deformation. On the other hand, in this $\mathrm{H} \neq 0$ case one is forced to take an infinite number of terms on the r.h.s. of either eq (9) [3] or eq (12) to close the conformal algebra, and get a consistent string theory. This is reminiscent of the case of closed string with closed string background around the "old fixed point", where the corresponding $2 \mathrm{~d} \sigma$ - model was shown to be nonperturbative nonrenormalizable in the weak field expansion, and an infinite number of counter terms was needed to preserve the worldsheet conformal symmetry. [8]. 


\section{Acknowledgement}

I would like to thank Pei-Ming Ho for many discussions. This research is supported

by National Science Council of Taiwan, R.O.C., under grant number NSC 89-2112-M-009045.The support from string study group of NCTS of Taiwan is also acknowledged. 


\section{REFERENCES}

[1] A. Connes, M.R. Douglas and A. Schwarz, " Noncommutative Geometry and Matrix Theory : Compactification on Tori," JHEP 9802, 003(1998), hep-th/9711162; M.R. Douglas and C. Hull , " D-branes and Noncommutative Torus," JHEP 02(1998)008, hep-th/9711165;C.S. Chu and P.M. Ho, "Noncommutative open String and D-brane," Nucl.Phys. B550 (1999) 151, hep-th/9812219," Constrained Quantization of open String in Background B Field and Non commutative D-brane", Nucl.Phys. B 568(2000)447, hep-th/9906192 ; V. Schomerus, "D-branes and Deformation Quantization," JHEP9906, 030(1999), hep-th/9903205.

[2] N. Seiberg, E. Witten, " String Theory and Noncommutative Geometry", JHEP 9909 (1999) 032, hep-th/9908142.

[3] P.M. Ho and Y.T. Yeh, " Noncommutative D-brane in non-constant NS-NS B field background" Phys. Rev. Lett. 85, (2000)5523, hep-th/0005159; P.M. Ho and S.P.Miao," Noncommutative Differential Calculus for D-brane in Non-constant B Field Background", hep-th/0105191.

[4] M. Green, J. Schwarz and E. Witten, "Superstring Theory", Cambridge Univ. Press, Cambridge, 1987.

[5] C.G. Callan, C. Lovelace, C.R. Nappi and S.A. Yost, " String Loop Corrections to Beta Functions," Nucl. Phys. B288,(1987) 525; A. Abouelsaood, C.G. Callan, C.R. Nappi and S.A. Yost, " Open Strings in Background Gauge Fields," Nucl. Phys. B280,(1987) 599; E.S. Fradkin and A.A. Tseytlin, "Nonlinear Electrodynamics Drom Quantized Strings," Phys. Lett. 163B,(1985) 123.

[6] J. Polchinski, Phys. Rev. Lett. 75 (1995) 4724; Phys. Rev. D50(1994) 6041. J. Dai, R.G. Leigh and J. Polchinski, Mod. Phys. Lett. A4(1989) 2073; R.G. Leigh, Mod. Phys. Lett. A4 (1989) 2767. 
[7] A. Cappelli, C.A. Trugenberger and G.R. Zemba, Nucl. Phys. B396, (1993)465; Phys. Lett. B306, (1993) 100; A. Cappelli, V.G. Dunne, C.A. Trugenberger and G.R. Zemba, Nucl. Phys. B398, (1993)531; S. Iso, D. Karabali, and B. Sakita, Phys. Lett. B296, (1992) 143; D. Karabali, Nucl. Phys. B419, (1994) 437; Nucl. Phys. B428, (1994) 531.

[8] S.R. Das and B. Sathiapalan, Phys. Rev. Lett. 57(1986)1511; Phys. Rev. Lett. 56(1986) 2664. 